\title{
Un ennemi « anonyme et sans visage »
}

Renseignement, exception et suspicion après le 11 septembre 2001

\section{Laurent Bonelli}

\section{(2) OpenEdition \\ Journals}

Édition électronique

URL : http://journals.openedition.org/conflits/1818

DOI : $10.4000 /$ conflits.1818

ISSN : $1777-5345$

Éditeur :

CCLS - Centre d'études sur les conflits lilberté et sécurité, L'Harmattan

Édition imprimée

Date de publication : 1 juin 2005

Pagination : 101-129

ISBN : 2-7475-8911-0

ISSN : 1157-996X

Référence électronique

Laurent Bonelli, « Un ennemi « anonyme et sans visage » », Cultures \& Conflits [En ligne], 58 | été 2005,

mis en ligne le 10 octobre 2005, consulté le 30 mars 2021. URL : http://journals.openedition.org/ conflits/1818; DOI : https://doi.org/10.4000/conflits.1818

Ce document a été généré automatiquement le 30 mars 2021.

Creative Commons License 


\section{Un ennemi « anonyme et sans visage »}

Renseignement, exception et suspicion après le 11 septembre 2001

\section{Laurent Bonelli}

1 La sanglante litanie des attentats perpétrés aux Etats-Unis le 11 septembre 2001, à Madrid le 11 mars 2004, et à Londres le 7 juillet 2005 a propulsé la lutte anti-terroriste au rang de priorité de l'ensemble des dirigeants des pays occidentaux. Les différents gouvernements ont de la sorte rivalisé de déclarations de fermeté et de propositions de réorganisation de leurs forces de sécurité, sommées de mieux coopérer et d'être plus efficaces dans leur lutte contre le radicalisme islamiste. Dans le même temps, de nouvelles structures de collaboration, d'échange ou de prospective virent le jour au sein des principales organisations internationales, ou intensifièrent leur activité. L'urgence et l'ampleur de cette « nouvelle menace » renforcèrent le rôle des services de renseignement, perçus comme les seuls à même de prévenir les conséquences de ce qui allait dès lors être qualifié d'« hyper-terrorisme ", de "nouveaux réseaux de terreur " ou de «terrorisme messianique». Nombre de mesures législatives et de restrictions des libertés furent d'ailleurs adoptées pour faciliter leur travail et assouplir les cadres juridiques qui l'encadraient.

2 Pour autant, l'observation des pratiques et des interactions au sein du petit monde des professionnels de l'anti-terrorisme amène à relativiser ce type de vision fonctionnaliste action/réaction, de même que les viriles invectives de nombre de ministres de l'Intérieur'. En effet, la lutte contre le «terrorisme » s'inscrit dans la durée, et résulte de multiples transactions entre les gouvernements, les agences de renseignement et les groupes clandestins, dans lesquelles chacun fait jouer ses intérêts politiques ou organisationnels, et tente d'imposer sa "vérité ». C'est ainsi que pour comprendre les mécanismes et les enjeux de cette lutte, il faut analyser la nature et l'évolution des relations d'interdépendances entre ces différents univers sociaux, en même temps que les contraintes - internes et externes - auxquelles ils sont soumis.

3 L'enquête présentée ici porte sur six services de renseignement. Deux français: Renseignements Généraux (RG) et Direction de la Surveillance du Territoire (DST); 
deux britanniques: Security Service (ex MI5) et Special Branch du Metropolitan Police Service de Londres (SO12), et deux espagnols : Comisaría General de Información (CGI) et Centro Nacional de Intelligencia (CNI), qui prend la suite en $2002 \mathrm{du}$ Centro Superior de Información de la Defensa (CESID) ${ }^{2}$. Elle visait à analyser les évolutions des missions des différents services après les attentats du 11 septembre aux Etats-Unis, et la manière dont ils percevaient l'islamisme radical. Elle repose sur des entretiens, de la littérature grise, des mémoires d'agents ou de directeurs des services, ainsi que sur une série de sources secondaires, comme des auditions et des rapports parlementaires ${ }^{3}$.

Elle n'est pas exhaustive, car définir les institutions en charge du renseignement est toujours problématique. L'indétermination fonctionnelle qui entoure leurs missions, leurs objectifs et leurs champs de compétences rend caduque toute tentative de clarification définitive. Les divisions institutionnelles des services: civil/militaire; interne/externe; politique/criminel; etc. s'avèrent le plus souvent fluctuantes et sujettes à de nombreux empiètements de territoires ${ }^{4}$. Des polices à statut militaire, comme la gendarmerie nationale française ou la guardia civil espagnole consacrent une bonne partie de leur activité au renseignement. Il en va de même pour les douanes, ou pour certains services liés à l'armée, comme la Direction Générale de la Sécurité Extérieure (DGSE), la Direction du Renseignement Militaire (DRM), ou la Direction de la Protection et de la Sécurité de la Défense (DPSD) en France, ou le Defense Intelligence Staff (DIS) en Grande-Bretagne.

5 Ce parti-pris est d'abord le fruit de contraintes pratiques : l'investigation a été menée dans le cadre d'une recherche collective financée par la commission européenne (ELISE, European Liberty and Security) d'une durée de trois ans qui ne permettait pas d'étendre le champ d'étude, a fortiori sur trois pays. Il résulte également du constat qu'en matière de radicalisme musulman, les services étudiés semblaient être les plus investis, tant en termes de moyens (humains et matériels) que de connaissances.

6 Cette enquête s'inscrivait d'emblée à rebours de toute une littérature qui fait des attaques du 11 septembre une sorte d'année zéro de la lutte anti-terroriste, suivant en cela les travaux de Graham T. Allison ${ }^{5}$, qui montrent ce que les visions institutionnelles des problèmes doivent à des routines organisationnelles qui leur préexistent et qui se réactivent à la faveur de situations nouvelles. Elle cherchait plutôt à percevoir les continuités et la permanence de certaines catégories d'analyse qui avaient été forgées sur d'autres terrains. Elle rompt de la sorte avec une approche mécaniste, qui verrait dans la transformation des missions des services de renseignement le simple fruit d'une " adaptation » à de nouvelles menaces. Elle insiste au contraire sur l'importance tant des jeux bureaucratiques et politiques, que de l'histoire incorporée de chaque institution dans l'élaboration de catégories d'appréhension de questions émergentes. Comme le rappelle Murray Edelman, les bureaucraties ont tendance « ̀̀ construire les problèmes comme justifications des solutions qu'elles proposent $»^{6}$. Ce qui concrètement signifie que la nature et la forme de ces tâches nouvelles résultent à la fois de transformations des contextes nationaux et international (et notamment de l'évolution des groupes clandestins et des formes de violence politique); des effets de la permanence des grilles d'analyse des services de renseignement et du travail perpétuel de relégitimation qu'ils vont mener auprès de certains secteurs de l'appareil d'Etat. 


\section{Autorités politiques, services de renseignement et régulation de la violence politique}

7 Les réactions des gouvernements aux attentats du 11 septembre 2001 et du 11 mars 2004 ne surgissent pas ex-nihilo de l'événement. Elles s'inscrivent dans une histoire longue et singulière des relations qu'ils entretiennent avec les différentes formes de violence politique auxquelles ils ont été confrontés, et dont il faut reconstituer l'économie générale.

D’abord, parler de "violence politique » et non de «terrorisme » permet d'éviter les impasses auxquelles conduit immanquablement l'usage de ce terme politiquement et moralement connoté. En effet, "terrorisme" ne décrit pas une réalité objective, qui s'imposerait à tous. L'armée allemande utilisait ce terme pour parler pour les résistants français, la Russie le fait pour les combattants tchétchènes et la Colombie pour les FARC, la Turquie pour le PKK, etc. Dans le même registre, Yasser Arafat était considéré comme un "terroriste ", jusqu'à ce qu'il devienne président de l'autorité Palestinienne, de même que le Front de libération national (FLN) algérien, avant d'accéder au pouvoir après l'indépendance. D'ailleurs, aucun groupe clandestin ne se revendique comme tel préférant selon les cas « combattants de la liberté », " nationalistes », " avant-garde du prolétariat ", " soldats de l'Islam », etc. L'apposition du label «terrorisme » - comme d'ailleurs celui de «bandit» - n'est de la sorte qu'un instrument ordinaire de délégitimation de certains mouvements et de leurs revendications, un instrument de négation de la nature politique du conflit ${ }^{7}$.

9 C'est notamment ce qui explique l'impossibilité d'arriver à une définition unanime dans le temps et dans l'espace du «terrorisme » et le traitement différentiel dont bénéficient les groupes clandestins. Il est intéressant à cet égard de noter que la liste «des personnes, groupes ou entités terroristes » définies par l'Union européenne - alors sous la présidence espagnole - après le choc du 11 septembre 2001, commence par une liste nominative d'individus soupçonnés d'appartenir à Euskadi Ta Askatasuna (ETA) ${ }^{8}$... Les négociations visant à établir ce genre de listes sont lourdes d'enjeux, en ce qu'elles engagent l'ensemble des signataires à lutter contre des groupes qui ne constituaient pas toujours des priorités, ou avec lesquels ils entretenaient des relations de nonagression. La position française sur la question basque a longtemps été l'objet de doléances de la part des policiers espagnols, au même titre que la tolérance britannique pour l'islamisme radical, de la part des services français. Chaque Etat définit de la sorte des modalités différentes de régulation de la violence politique. Celles-ci intègrent successivement ou simultanément non-intervention, négociation, pression, répression policière, voire action militaire.

10 La forme de ces réactions d'Etat dépend à la fois de l'histoire de ces relations, des évolutions des rapports de forces politiques entre les parties en présence et de l'évaluation de l'ampleur de la menace que font peser les mouvements clandestins sur l'ordre social et politique.

11 C'est ainsi qu'en France, la violence des groupes nationalistes corses, pourtant responsables de centaines d'attentats à l'explosif (l'île en a connu 1815 entre 1993 et 1998), visant des bâtiments publics (trésoreries, locaux des douanes et des impôts, etc.), voire les services de police et de gendarmerie (les locaux de la brigade de Pietrosella ont par exemple été totalement détruits par une attaque en 1997) ne déclenche pas les 
mêmes interventions policières et judiciaires que celle liée aux groupes radicaux musulmans ${ }^{9}$. C'est aussi en vertu de l'histoire - coloniale et post-coloniale - que les services français ont longtemps accordé plus d'attention aux groupes islamistes armés engagés dans le conflit algérien qu'aux autres. Ceci transparaît dans les propos de Pierre de Bousquet de Florian, actuel directeur de la Direction de la Surveillance du Territoire (DST) quand il expliquait en septembre 2002 que :

«La France ne paraît pas être la priorité de Al-Qaida. (...) Le danger, c'est qu'il y a aujourd'hui un vrai rapprochement entre le groupe salafiste pour la prédication et le combat [GSPC] qui agit en territoire algérien et les gens d'Al-Qaida. (...) Ce rapprochement peut constituer une menace : l'amalgame mêlant une vieille idée de la France coloniale, la mauvaise opinion qu'ont un certain nombre de jeunes Français d'origine maghrébine de la situation au Proche Orient, une éventuelle guerre contre l'Irak est potentiellement explosif $»^{10}$.

De la même manière, au moins jusqu'aux attentats du 11 mars 2004, les services de renseignement espagnols restaient en pratique largement focalisés sur ETA, qu'ils tiennent pour responsable de 817 assassinats - dont 478 au sein des forces armées ou des corps de police - entre 1968 et 2003, et de plusieurs centaines d'attentats. Les chiffres sont d'ailleurs éloquents, puisque sur les 500 agents de la Comisaría General de Información (CGI), un dixième seulement s'occupait de «terrorisme international». C'est aussi ce qui explique en partie que les soupçons aient été initialement dirigés vers l'organisation basque. Comme l'indiquait rétrospectivement Ignacio Astarloa, secrétaire d'Etat à la sécurité du gouvernement du parti populaire : « ce jour là, ceux que nous craignions réellement, c'était ETA $»^{11}$.

Les postures que vont adopter les différents gouvernements face à des actions radicales dépendent de la sorte largement de l'histoire de la régulation de la violence politique et de l'évaluation qu'ils font de la vulnérabilité présente.

Elles sont également contraintes par la nécessité dans laquelle se trouvent les autorités politiques de rassurer leurs concitoyens, pour éviter le déclenchement de ce qu'elles nomment la " psychose terroriste ». La lutte anti-terroriste s'inscrit en effet dans des temporalités longues et demeure par définition discrète, puisqu'il s'agit de reconstituer des réseaux et d'en comprendre le fonctionnement. Elle s'accompagne donc souvent de mesures visibles - et fortement publicisées - qui montrent que l'on fait quelque chose en matière de terrorisme. En France, l'activation du plan Vigipirate à son niveau maximum après le 11 septembre 2001 répondait à cette double logique de prévention d'actes terroristes et d'affichage de la réponse politique. Le déploiement de militaires et la multiplication des contrôles dans le métro, dans les gares ou les aéroports, visait à rendre plus difficile un potentiel attentat, mais surtout montrait que l'Etat avait pris au sérieux la menace et déployait des moyens pour y répondre. Peu importe, d'ailleurs, si les résultats de cette opération se sont davantage traduits par l'augmentation des arrestations d'étrangers en situation irrégulière que par un quelconque succès en matière anti-terroriste. Le déploiement de chars à l'aéroport londonien d'Heathrow, en février 2003, procède de la même dynamique.

15 La relation terroriste se définit de la sorte par une interdépendance stratégique (un "échange de coups ", cher à Thomas $\mathrm{C}$. Schelling ${ }^{12}$ ) entre gouvernements et groupes clandestins, arbitrée par la publicité de certains actes et déclarations de chacun des acteurs. Les annonces de fermeté font de la sorte écho à la violence spectaculaire (les attentats par exemple), alors même que les modalités de sortie de crise reposent autant sur l'action policière que sur le maintien ou la reconstruction d'un dialogue impliquant 
des engagements réciproques. Des mesures discrètes de clémence pour des activistes emprisonnés à la modification d'une posture politique ou diplomatique, le spectre des dispositions pour ramener le calme est très vaste. Et c'est autant la subtilité de cet équilibre que le niveau auquel se prennent ces décisions politiques qui expliquent qu'au-delà des déclarations volontaristes sur la coopération internationale, les problématiques nationales priment largement ${ }^{13}$.

16 Pour autant, cette relation s'étend au-delà du triptyque gouvernements / groupes clandestins / opinion publique, souvent évoqué. Elle inclut également les services de renseignement, dont on aurait tort de penser qu'ils ne sont qu'un simple instrument entre les mains des autorités politiques. La question de ces relations occupe une place importante dans la littérature consacrée au sujet, qu'elle soit savante, journalistique ou indigène. Les interprétations balancent entre un pôle "dénonciateur ", qui fait des services de surveillance un véritable Etat dans l'Etat et un pôle « instrumental », leur assignant un rôle de simple outil au service de la décision politique. Les tentatives les plus intéressantes pour dépasser ces oppositions et rendre compte des évolutions historiques de la place des services au sein d'un même Etat proviennent de travaux comme ceux de William W. Keller ou de Peter Gill ${ }^{14}$. Ce dernier propose par exemple de saisir les relations entre Etat et services de renseignement au travers des notions d'autonomie et de pénétration. L'autonomie renvoie à l'indépendance des agences vis-àvis des influences extérieures pour ce qui concerne leurs politiques et leurs pratiques; la pénétration concerne la variété des techniques par lesquelles elles contrôlent et surveillent les autres agences et la société en général. L'interaction entre ces deux axes permet ainsi de définir des types idéaux de relations, qui vont de l'Independant Security State (forte autonomie et pénétration)au Domestic Intelligence Bureau (faible autonomie et pénétration), en passant par la Political Police (autonomie et pénétration moyennes). Pour autant, si ces travaux permettent de penser des formes d'autonomies institutionnelles, ils restent prisonniers d'une vision trop unifiée de l'Etat, qu'ils ne pensent pas comme des groupes dotés de formes de ressources symboliques particulières, en concurrence pour dire et faire l'Etat au nom de l'Etat. Comme l'indique Bernard Lacroix : "l'Etat n'existe que sous l'espèce des luttes dans l'Etat, mais aussi autour de l'Etat pour la maîtrise de son autorité et de son crédit $»^{15}$. Et c'est justement dans ces luttes que va s'inscrire et se légitimer le travail de renseignement.

En effet, l'anti-terrorisme ne constitue pas la seule source de légitimité des services de renseignement. Celle-ci est avant tout la contrepartie de la production d'informations "pertinentes» dans le champ politique, à l'aune desquelles on va jauger leurs performances et leur "utilité ». Si dans le spectre de leurs activités, les priorités varient selon les contextes et les configurations locales, les services de renseignement collectent toutes les informations qu'ils pensent pouvoir être - un jour - utiles aux autorités. Leur travail consiste de la sorte à fournir aux gouvernements des renseignements permettant d'anticiper l'apparition et l'évolution d'une crise, ou plus largement de savoir ce qui se passe, se dit et se fait dans certains secteurs déterminés de la vie sociale et politique. Cette production de ressources dans le jeu politique intérieur vaut également dans les relations internationales, où elles peuvent servir de monnaie d'échange ou de gages de bonne volonté. Comme l'expliquait l'un de mes interlocuteurs des Renseignements Généraux :

«On s'occupe également de groupes qui pourraient porter atteinte aux relations diplomatiques de la France. C'est ainsi par exemple que sur pression du Quai d'Orsay, qui répondait lui même à des pressions des autorités péruviennes, on a mis 
le paquet sur les militants de Tupac Amaru en France, lors de la prise d'otages de l'ambassade du Japon à Lima [décembre 1996]. On les a surveillés, écoutés, on est allé leur parler, etc. C'est la même chose lors de la venue à Paris du président iranien [octobre 1999]. On savait parfaitement qu'il ne courrait aucun danger de nature terroriste. Ça n'a pas empêché (...) une rafle de grande ampleur chez les opposants au régime. Ce sont des mesures à très forte visibilité politique, rien d'autre... Mais c'est important ». [Entretien, mars 2000]. concernent également une partie de l'opposition et la presse. De ce point de vue la politique des fuites constitue un mode ordinaire de légitimation des services de renseignement auprès d'agents sociaux officiellement non destinataires de leurs informations. Le secret qui entoure ces dernières renforce considérablement leur force, en leur conférant une "vérité » qui n'a rien à voir avec leur véracité ou leur justesse. C'est avant tout leur qualité de ressource protégée dans le champ politique qui fonde leur autorité. Comme le signale Alain Dewerpe: «Dans le régime d'opinion publique, l'usage du secret s'inscrit, en le révélant, dans le rapport entre des agents qui y possèdent les compétences d'une participation active et ceux qui, dépourvus de compétences, sont réduits à une participation passive; et l'état actuel du champ, en organisant ces relations, renvoie aux modes de structuration de l'accès au savoir politique et, partant, à la mise en réserve de ce savoir, en d'autres termes, à la genèse historique du secret comme forme et enjeu de la domination politique dans le régime de la publicité contemporaine $»^{16}$.

Cette légitimité leur confère une autonomie organisationnelle, qui se traduit par un renoncement - implicite le plus souvent - du personnel politique au contrôle au jour le jour de leurs activités. L'absence de commissions parlementaires de contrôle (en France), ou les multiples dérogations qui limitent leur action (en Grande-Bretagne et en Espagne) sont éloquentes à cet égard. Cette autonomie relative définit l'espace d'activité des services de renseignement, bien au-delà de leurs missions « officielles ». En effet, leur rôle d'intermédiaires entre les pouvoirs institués et groupes contestataires ne se borne pas, loin s'en faut, à celui de simples exécutants. Le travail de prévention, d'anticipation, de négociation, voire dans certains cas de déstabilisation qu'ils conduisent influe tant sur les stratégies de ces groupes que sur celles des autorités. Les services de renseignement ne se limitent pas toujours à la collecte d'informations. De la disqualification publique de certains groupes, au sabotage de leurs actions ou à la destruction de leurs leaders - morale, symbolique et dans certains cas physique - en passant par la démoralisation des militants ou l'exacerbation des tensions internes, les stratégies visant à contrer des mouvements sociaux ou politiques sont nombreuses ${ }^{17}$. Dans le cas des Renseignements Généraux français, ce fut même l'objet exclusif de certaines sections, aux méthodes discrètes et parfois illégales, comme la section manipulation, le groupe des enquêtes réservées, ou de cellules opérationnelles plus informelles ${ }^{18}$. En Espagne, il faut rappeler l'épisode des Groupes Anti-terroristes de Libération (GAL), qui assassinèrent, avec la complicité du CESID et de la guardia civil, des réfugiés basques en France à la fin des années 1980, ou en GrandeBretagne, les polémiques autour de la participation des services de renseignement - et notamment de la Force Research Unit (FRU) - à l'élaboration des pratiques de shoot to kill destinées à éliminer des militants présumés de l'IRA ${ }^{19}$.

Les services de renseignement occupent de la sorte une place particulière dans la structuration et la régulation des échanges politiques au sein même des régimes démocratiques. Ils sont à la fois un vecteur - partiellement autonome - de l'exercice de 
la violence d'Etat et des gardiens de l'ordre politique. Par leurs activités, ils participent en effet à la structuration de l'économie générale de la contestation, ce qui fait d'eux des agents de la gestion négociée des règles du désordre ${ }^{20}$. Plus encore, par les informations qu'ils collectent, les catégorisations et les mises en forme qu'ils opèrent, ils participent à la clôture du jeu politique. En effet, en qualifiant ou disqualifiant certains groupes, sur la base de leurs propres appréciations, les services de renseignement leur permettent ou non d'y participer. Il importe donc de s'intéresser aux principes de production de leurs visions du monde social, historiquement et institutionnellement constituées.

\section{Missions, logiques et savoirs des services de renseignement}

21 Au-delà de leurs différences nationales et de priorités, les agents des services de renseignement partagent certaines manières de percevoir le monde social. Ceci pourrait surprendre, compte tenu de l'hétérogénéité de leurs statuts et de leurs compétences : certains sont policiers (Renseignements Généraux [RG] et Direction de la Surveillance du Territoire [DST] en France, Comisaría General de Información [CGI] en Espagne, Special Branch en Grande-Bretagne), alors que d'autres proviennent des universités ou des écoles militaires (Security Service en Grande-Bretagne, partie du Centro Nacional de Intelligencia [CNI] en Espagne) ; certains ont des aptitudes judiciaires qui leur permettent de mener des opérations coercitives (DST, Special Branch et CGI), quand d'autres limitent généralement leur activité à la collecte et à l'analyse d'informations (RG, Security Service et CNI). L'ensemble de ces différences, reposant sur des socialisations professionnelles distinctes plaide pour une variété de matrices de perception, d'appréciation et d'actions irréductibles les unes aux autres.

Et pourtant. L'ensemble de ces professionnels partage une croyance fondamentale dans le renseignement, c'est-à-dire dans un certain nombre d'enjeux fondamentaux liés à l'existence même de l'activité. Ceci implique une illusio commune, (ie. la croyance que le jeu vaut la peine d'être joué), souvent perçue négativement par leurs collègues d'autres services $^{21}$, de même qu'une complicité objective sous-jacente à tous les antagonismes (un accord sur les désaccords).

23 Le renseignement est en effet un champ partiellement autonome, qui impose aux agents qui y sont engagés la détention d'une forme de capital spécifique ${ }^{22}$. Ce capital consiste en des savoir-faire, des techniques et en un ensemble de croyances propres, historiquement constitués et sédimentés dans les services, qui se transmettent aux nouveaux entrants et sont la condition de leur participation au jeu.

Il se traduit par la place centrale qui est accordée aux rapports de forces politiques et suppose que les systèmes de perception et les principes d'action des agents de renseignement s'opposent à ceux issus du monde judiciaire, pour qui le rapport au droit (à la loi) est central. Des autorités de tutelle (gouvernement/magistrat) aux cibles de l'activité (politique/criminel), en passant par les modes opératoires (prévention et pro-activité/répression), tout sépare ces deux activités. Même dans le cas où ces agents sont des policiers, on s'aperçoit que ce qui les éloigne de leurs collègues des services de police judiciaire ou des polices urbaines est bien plus qu'une différence de missions. Les travaux que j'ai menés en France sur les carrières des membres des Renseignements Généraux montrent de la sorte une remarquable permanence dans l'institution et un faible taux de passage vers les autres métiers policiers ${ }^{23}$. Ils vont ainsi intérioriser des 
univers de pratiques et de routines bureaucratiques qui se caractérisent par un intérêt pour le jeu politique, une maîtrise pratique de ses enjeux et par un attachement à l'ordre légitime et à sa conservation. Ces dispositions antisubversives sont une importation des logiques du soupçon, propres aux policiers en général ${ }^{24}$, dans l'activité politique et expliquent la récurrence des visions en termes de "complot» et de " manipulation».

L'une des manifestations les plus claire de ces dispositions est le degré d'organisation que les services de renseignement prêtent à leurs adversaires dans leurs rapports et leurs synthèses. Ils ont tendance à voir derrière la moindre initiative locale un élément d'une stratégie politique globale, et à considérer des groupes ou des individus autonomes comme les agents d'une organisation occulte et structurée. Yves Bertrand, (directeur des Renseignements Généraux français de 1992 à 2004) commentait de la sorte la surveillance des discours tenus dans les mosquées :

« Nous avons, par exemple, été particulièrement vigilants après les attentats du 11 septembre 2001. Nombre d'observateurs s'attendaient à des dérapages nombreux (...) curieusement, les responsables des mosquées et des associations ont très bien contrôlé leurs troupes; ce qui, d'ailleurs, n'est pas pour nous rassurer. Cela signifie que le tissu associatif, qui est très dense, fonctionne à merveille (...) que la communauté est très bien contrôlée par ces associations ${ }^{25}$.

Dans ce type d'analyse, le calme est pire que la tempête, car il dissimulerait quelque chose de plus inquiétant. Au prisme de cette vision menaçante du monde et de la prédominance donnée au caché sur le visible, la réprobation quasi unanime de ces actes sanglants devient ainsi une préoccupation supplémentaire, qui constitue une nouvelle justification de leur travail de surveillance. Parlant de la contre-subversion aux EtatsUnis, Michael Rogin montre la double fonction de ses discours inquiets : «donner corps à ses propres angoisses tout en y trouvant l'occasion de donner libre cours à des désirs interdits. En effet, la diabolisation de ses adversaires vient en quelque sorte légitimer l'usage par elle des armes mêmes qu'elle leur attribue, mais au nom, cette fois de la nécessité supérieure de mettre en échec les plans de la subversion ${ }^{26}$. Cette rivalité mimétique explique la surévaluation systématique des capacités de leurs adversaires, et les plaidoyers constants pour la limitation des libertés individuelles et collectives, censées les favoriser. C'est ainsi que les services de renseignement opèrent la confusion perpétuelle entre le techniquement possible et le socialement probable. Par exemple, les risques d'utilisation d'armes de destruction massive (nucléaires, chimiques ou bactériologiques) par des groupes radicaux n'apparaissent pas sous leur regard comme une éventualité soumise à conditions (politiques notamment), mais comme la prochaine étape. Jorge Dezcallar de Mazarredo, directeur du CNI jusqu'en 2004, expliquait de la sorte : "maintenant, rien n'est comme avant. Nous sommes devant un nouveau Hiroshima en puissance, avec la différence que n'importe quel fanatique illuminé peut appuyer sur le bouton ${ }^{27}$.

Ces visions découlent autant des dispositions spécifiques des agents de renseignement, et de leurs routines bureaucratiques que d'une volonté de "grandir " la menace, qui assure de fortes rétributions matérielles et symboliques. C'est ce que signalait un ancien directeur de la DST au sujet de l'Islam :

« Le contre-espionnage classique était la raison de vivre de la DST. Les agents de ces services ont cherché à se reconvertir en trouvant une menace aussi globale que le communisme, et c'est ainsi qu'on est passé des intégrismes à la menace islamiste ». [Entretien, avril 2000]. 
Il ne s'agit pas pour autant de cynisme. Les agents des services de renseignement ressemblent à bien des égards au magicien décrit par Marcel Mauss, qui «ne peut pas être conçu comme un individu agissant par intérêt, pour soi et par ses propres moyens, mais comme une sorte de fonctionnaire investi par la société, d'une autorité à laquelle il est engagé à croire lui-même. (...) Il a tout naturellement l'esprit de sa fonction, la gravité d'un magistrat ; il est sérieux, parce qu'il est pris au sérieux et il est pris au sérieux parce qu'on a besoin de lui $»^{28}$.

Ils sont d'autant plus pris au sérieux qu'ils appartiennent à un champ de production restreint qui a le quasi-monopole de la production de cette forme particulière de bien symbolique qu'est l'interprétation de la «menace». Le secret qui entoure les informations qu'ils obtiennent et produisent constitue en effet une ressource protégée, garantie par une position d'autorité dans l'Etat, qui ne s'expose qu'au jugement de leurs pairs. Ce qui les place d'emblée hors de l'alternative du vrai et du faux. Et ce n'est pas tant la pertinence de leurs analyses qui fonde leur validité auprès de certains secteurs du monde politique et des médias que les flux transactionnels de longue durée qu'ils entretiennent avec ces univers. C'est également la raison pour laquelle la réfutation fréquente par les faits de leur vision apocalyptique du monde n'ébranle pas la confiance qui leur est portée. Confiance, qui vient en retour légitimer et durcir leurs modes d'interprétation.

Si les agents des services de renseignement partagent des formes de conceptions communes du monde, ils n'en sont pas moins engagés dans des luttes pour conserver ou subvertir les rapports de force au sein du champ du renseignement. C'est ainsi que les actions - notamment violentes - des groupes clandestins constituent autant d'occasions de réévaluer la place et l'importance de chaque agence en faisant valoir ses propres analyses. En France, la DST, qui a de plus en plus de fonctionnaires implantés à l'étranger et assure des missions de contre-espionnage, a tendance à relier systématiquement tout acte terroriste à des commanditaires étrangers, là où les RG cherchent des causes et/ou des réseaux plus hexagonaux. L'affaire Khaled Kelkal est particulièrement emblématique de ce phénomène. Ce jeune Français de Vaulx-en-Velin fut impliqué dans les attentats de 1995, avant d'être abattu par les gendarmes. La DST en fit un agent de réseaux étrangers opérant sur le territoire (le GIA algérien), alors que les RG tentèrent de resituer l'affaire dans le cadre de la cité où elle est née, pour montrer les risques de radicalisation de jeunes Français issus de l'immigration. Au-delà des principes de production de ces analyses, les concurrences bureaucratiques sont réelles. Il en va de la légitimité des services, voire de leurs budgets de fonctionnement.

Dans le cas des réseaux islamistes radicaux trans-nationaux, ce type d'oppositions se reproduit. La DST va insister sur le caractère "international » de Al-Qaida et les liens entre des groupes disséminés dans différents Etats, alors que les RG vont davantage s'intéresser aux risques qui pèsent sur les communautés musulmanes françaises. Comme le soulignait l'un de mes interlocuteurs :

« Il existe à la fois une proximité géographique et une proximité d'origine entre les groupes à risques et les jeunes des banlieues. Leur instrumentalisation est limitée, mais elle existe. Elle nous préoccupe à plusieurs niveaux : d'abord, ces groupes se financent par des trafics, des vols, bref du droit commun dans lesquels sont impliqués des jeunes des cités. Ensuite, ceux-ci récupèrent une partie du discours anti-français des islamistes, qui pourrait à terme permettre le développement de groupes terroristes autonomes, qui n'existent toutefois pas aujourd'hui. Enfin, nous sommes préoccupés par la diffusion du savoir-faire terroriste dans les banlieues. Les stages que suivent certains gamins, en Bosnie ou ailleurs, les publications qui 
circulent, etc. leur apprennent comment fabriquer des bombes ou des cocktails Molotov, au point que de plus en plus de jeunes savent comment faire...". [Entretien, avril 2000]. leaders et des associations religieuses ne date pas des attentats contre le World Trade Center et le Pentagone. Elle constitue l'une des routines du contre-espionnage. En effet, les services de renseignement d'un certain nombre de pays d'émigration ont depuis longtemps utilisé les infrastructures religieuses pour contrôler leurs exilés. C'est particulièrement vrai en France pour les services marocains et algériens, mais ça vaut également en Espagne. Comme l'indiquait Jorge Dezcallar de Mazarredo, ancien directeur du CNI, au sujet des prêches donnés dans les mosquées de son pays :

"C'est quelque chose que nous suivons avec attention. (...) Les mosquées sont un champ d'information privilégié pour des pays tiers, qui tentent de les pénétrer pour contrôler les colonies d'immigrants de certaines nationalités. Nous savons quels sont les plus agressifs et quels sont les plus religieux. Ou ceux qui interfèrent dans les sujets politiques, puisqu'il y en a également $»^{30}$.

Ce type de surveillance va donc se développer à mesure que grossissent les communautés en provenance notamment des anciens empires coloniaux. En France, il 
est lié au processus de sédentarisation progressive des travailleurs immigrés (en partie dû à l'arrêt officiel de l'immigration de travail en 1974). En Grande-Bretagne, il vient de l'afflux de réfugiés pakistanais, ou de frères musulmans pourchassés dans les anciennes colonies britanniques qui donnent naissance, à la même période, au Londonistan. En Espagne, le processus est plus récent, même si l'université de Grenade, qui accueille depuis de longues années des étudiants en provenance d'Afrique du nord, a toujours été sous surveillance.

Mais l'intérêt des services de renseignement pour les communautés musulmanes va prendre une tournure assez différente à la suite d'un certain nombre d'évènements internationaux liés à l'islamisme politique. En France, les principales étapes du développement des sections spécialisées ont été la révolution iranienne de 1979, la situation au Moyen Orient et les attentats du réseau Fouad Ali Saleh de 1985-1986 et surtout la situation algérienne après juin 1991, qui devait déboucher sur une nouvelle vague d'attentats en 1995. Ils vont de la sorte concentrer leur attention sur les activités des groupes clandestins musulmans (les groupes islamistes armés [GIA] algériens surtout), que ce soit pour contrer leurs velléités d'actions violentes sur le territoire, ou pour perturber le travail logistique qu'ils peuvent y mener (propagande, recrutement, circuits de financement, etc.).

Les attentats du 11 septembre 2001 aux Etats-Unis, du 11 mars 2004 en Espagne et ceux du 7 juillet 2005 en Angleterre ont modifié brutalement les modes historiquement constitués de régulation de la violence politique, auxquels les services de renseignement étaient habitués.

C'est d'abord l'irruption brutale en temps de paix, sur le territoire d'Etats occidentaux d'une violence létale ayant occasionné un nombre de victimes inconnu jusqu'alors. Ces massacres créaient un effet de seuil sans commune mesure avec les actions radicales antérieures. Mais au-delà de leur ampleur, ces attentats subvertissent le nomos - c'està-dire le principe de vision et de division fondamental qui est caractéristique de chaque espace social ${ }^{31}$ - du champ politique. Les groupes qui en sont à l'origine ne s'appuient pas sur des bases et des revendications nationalistes, ou de classe. Ces deux schèmes de classement structuraient largement la violence politique dans les démocraties occidentales, et continuent de le faire dans de nombreux conflits, y compris dans ceux impliquant des mouvements radicaux musulmans (en Palestine, ou en Tchétchénie par exemple). Les mouvements se réclamant de Al-Qaida ne semblent en effet avoir d'autres velléités que la construction de ce que Farhad Khosrokhavar appelle une néoumma guerrière, reposant sur « la haine de l'occident, la mythification de la communauté islamique d'origine, la volonté de restaurer l'islam dans sa splendeur de jadis et la promotion de la mort en martyr dans le djihad ${ }^{32}$. Cette incertitude quant aux finalités ressort clairement dans les propos d'Eliza Manningham-Buller, directrice générale du Security Service, quand elle explique que :

« Nous sommes entrés dans une nouvelle période de l'histoire du terrorisme. Moins régionale et nationale, cette nouvelle phase est caractérisée par un terrorisme suicidaire, des attentats menés par des terroristes qui cherchent à infliger volontairement des dommages massifs à des civils et qui appartiennent à des groupes qui n'ont aucun intérêt à la négociation $"^{33}$.

Cette transformation des cadres mentaux de la mobilisation pose aux services de renseignement des difficultés intellectuelles pour comprendre - et pouvoir anticiper des actions qui répondent à d'autres logiques que celles auxquelles ils étaient accoutumés. Non seulement, les groupes qui en sont responsables semblent faire fi des 
traditionnels processus politiques de négociation, mais leur autonomie, tant tactique que stratégique, et leur absence de base territoriale ou sociale compliquent largement la construction de cet échange. A la différence d'autres groupes clandestins, pour lesquels ils avaient des interlocuteurs identifiables - liés aux mouvements eux-mêmes, à des vitrines politiques, ou à des gouvernements leur apportant leur soutien - ce type de radicalisme musulman apparaît pour les agences de renseignement comme un " ennemi anonyme et sans visage " $^{34}$. La lutte anti-terroriste tend de la sorte à se limiter à sa dimension coercitive, les agences de renseignement voyant dans la neutralisation des réseaux le seul moyen d'empêcher leur passage à l'acte. De là, les plaidoyers pour la mise en place de moyens d'exception, qu'ils soient policiers ou judiciaires, et l'attention soutenue portée aux communautés musulmanes nationales, qui restent toujours suspectes de faire primer une identité «islamique » sur une identité nationale et de constituer une « cinquième colonne » du terrorisme.

41 Les services de renseignement vont d'abord contrôler des fonctions ou des lieux. En utilisant des méthodes de travail dites de "milieu fermé» (écoutes, filatures, traitement d'agents, etc.), ils vont ainsi surveiller les mosquées, les prêches, les associations culturelles, etc., c'est-à-dire tous les espaces où ils pensent pouvoir trouver des «intégristes». Comme l'indiquait l'un de mes interlocuteurs de la direction centrale de Renseignements Généraux :

«Notre travail consiste à faire du travail opérationnel sur des gens dans des mosquées. (...) On nous reproche d'avoir une vision sécuritaire de l'Islam. Ce que je dis toujours, c'est je ne suis pas musulman. Je n'ai pas étudié la culture musulmane. Je la connais un peu, empiriquement par mon travail. Je suis donc incapable de juger ce qu'est un bon musulman. (...) Que les musulmans pratiquent leur religion, c'est normal et c'est très bien. Par contre, je suis capable de dire si des gens représentent un trouble à l'ordre public. Ça c'est mon métier. Et comme un acte terroriste doit être commandé par une Fatwah, délivrée par quelqu'un d'habilité à le faire, on s'intéresse à ceux qui ont ce pouvoir ». [Entretien, avril 2000].

Ils vont de la sorte être attentifs à l'activité des institutions et des groupes musulmans, ainsi qu'à leurs rapports de force internes et à leurs évolutions. Pour les services de renseignement, c'est en effet la capacité « de se cacher en pleine lumière, d'être vu mais pas remarqué $»^{35}$, qui caractérise les membres de ces groupes radicaux. Il s'agit alors de repérer qui, dans les communautés musulmanes, est susceptible de rejoindre leurs rangs. Ce travail va passer par l'élaboration de " profils », de « figures » typiques. C'est l'objet des méthodes proactives,qui visent «à partir de corrélations statistiques établies sur des trajectoires individuelles, à produire un dispositif d'information orienté vers l'action répressive, capable d'anticiper en amont de la commission d'une infraction ou d'un délit, le comportement probable d'individus aux propriétés similaires $»^{36}$. Fondant leur travail sur des études de cas d'individus impliqués dans des actions clandestines, les services de renseignement vont élaborer des trajectoires sociales modales et apporter une vigilance particulière aux activités de ceux qui répondent à ces propriétés. C'est ainsi qu'une origine étrangère (et particulièrement d'un pays musulman), un niveau d'études relativement élevé, la fréquentation de telle ou telle association ou mosquée ("fondamentaliste» ou "salafiste» surtout), des voyages fréquents, une rupture professionnelle et des séjours à l'étranger (à Londres notamment), etc. déclenchent presque automatiquement l'attention des agences de renseignement.

43 La suspicion est encore plus grande pour les «convertis ». Cette figure de l'européen rallié à l'Islam concentre l'ensemble des propriétés de la «dangerosité », telle que les 
services de renseignement la définissent. Comme l'indiquait Yves Bertrand, ancien DCRG :

«La dangerosité, (...) c'est le clandestin. Le clandestin, ce sont les réseaux. Je rappelle que la France a été le premier pays touché par les attentats terroristes au milieu des années 1990. Nous avons découvert à cette occasion (...) le phénomène des convertis (...) et leur importance au sein de ces réseaux. Les convertis jouaient et jouent toujours le rôle que jouaient les Français qui étaient dans le Front de libération nationale [FLN]; les 'porteurs de valise', comme on les appelait. En fait, ils étaient bien plus importants que de simples porteurs de valise. J'établis cette comparaison non pas sur un plan idéologique mais pour montrer comment cela fonctionne $\aleph^{37}$.

Ce n'est pas tant le potentiel de radicalité des convertis - lié à la conversion récente et aux surenchères qui peuvent l'accompagner - qui inquiète les membres des services de renseignement que leur appartenance à la communauté nationale, dans laquelle ils se fondent. L'image des porteurs de valise - outre qu'elle montre la persistance des schémas issus de la colonisation - est intéressante, puisqu'elle devient la métaphore de l'ennemi invisible, qui bénéficie de tous les avantages que procure la nationalité (libre circulation, protection juridique, facilités administratives, etc.), et les retourne contre les intérêts de l'Etat qui les lui a concédés.

En même temps qu'ils essaient de neutraliser les membres des groupes radicaux, les services de renseignement tentent de démanteler leurs réseaux de soutien, qu'ils supposent être implantés dans les quartiers comportant une forte proportion d'immigrés, en provenance de pays majoritairement musulmans. C'est ainsi, par exemple, qu'ils surveillent les proches des individus soupçonnés de prendre part à des actions radicales. Comme l'expliquait Pierre de Bousquet de Florian, directeur de la DST :

« Nous travaillons aussi beaucoup sur les entourages. Certains activistes, sans jamais avoir habité en France ou avec peu de liens ici, peuvent y avoir des attaches: un cousin ou un copain, une sœur et un beau-frère. Sans parler de réseau organisé, ils peuvent bénéficier de complicités plus ou moins volontaires, de solidarités amicales ou familiales susceptibles de déboucher sur des aides logistiques ${ }^{38}$.

On retrouve ici des figures classiques du travail de contre-subversion, qui déduit mécaniquement des systèmes d'attitudes de proximités sociales, affectives ou culturelles et en fait le motif de la surveillance ${ }^{39}$.

Or, il importe d'être particulièrement prudent. La situation de disqualification sociale dans laquelle sont un certain nombre de jeunes des quartiers populaires des grandes métropoles occidentales les conduit à reconstruire et à «bricoler» des identités religieuses notamment - visant à restaurer des formes de dignitét ${ }^{40}$. Leur apparente radicalité (dans le discours notamment) n'a pourtant rien à voir avec celle des groupes clandestins. De même, les travaux sur des individus qui sont entrés dans l'action violente insistent sur la singularité des trajectoires et des histoires de vie plutôt que sur une " carrière » systématique ${ }^{41}$. Le profilage et les interprétations que celui-ci autorise font fi des modalités et des motivations différentielles de l'engagement religieux et agrègent cette hétérogénéité en une même catégorie "menaçante». Ils jettent le soupçon sur une bonne partie de la communauté musulmane, en reliant tout élément, fait ou activité culturelle et religieuse à la potentialité terroriste. Al-Qaida, l'image d'une religion conquérante, homogène et guerrière, menaçante pour 
l'occident, construite dans certains cénacles stratégistes ${ }^{42}$ et la montée en puissance (même si elle reste relative) des revendications liées à l'exercice du culte musulman et le renouvellement des organisations religieuses, au sein des pays occidentaux ${ }^{43}$. Ces glissements vont accréditer simultanément les thèses de la dangerosité extrême de l'islam et jeter le doute sur la loyauté des communautés d'immigrés, censées fonctionner comme des «réservoirs terroristes». Ils autorisent de la sorte des généralisations hâtives. Ainsi, le commissaire français Richard Bousquet affirme par exemple que : «le vivier humain que peuvent fournir nos quartiers en difficulté à l'islamisme radical est toujours aussi grouillant de 'beurs' réislamisés et de convertis frottés de délinquance prêts à se lancer dans l'aventure terroriste au signal d'un cerveau du djihad international $\aleph^{44}$. Cette suspicion généralisée pour les étrangers n'est pas sans rappeler le mythe de la cinquième colonne $\mathrm{e}^{45}$ et de l'ennemi intérieur. Elle déborde même largement le cadre du terrorisme, pour devenir une menace à la cohésion nationale.

La lutte contre la "menace terroriste " ne borne pas le travail de surveillance des services de renseignement. Dans le cas français, ils portent également une attention particulière aux groupes musulmans prosélytes, tels que la Jama'a at-Tabligh, par exemple. Par des écoutes téléphoniques, ils savent que certains de leurs militants ont reçu des injonctions les enjoignant de prêcher dans les quartiers populaires, sur le thème de l'exclusion sociale et économique des adolescents qui s'y trouvent. Ce que confirment leurs observations. Ils constatent également le rôle que peuvent jouer certains groupes ou leaders religieux dans l'apaisement des tensions d'un quartier et l'influence modératrice qu'ils peuvent avoir sur les comportements "déviants » des plus jeunes. Ne pouvant toutefois mesurer l'impact direct de ce discours sur les adolescents et les jeunes adultes des quartiers populaires, ils vont recourir à la notion vague de «communautarisme» (parfois d'«arabité»), censée menacer l'unité républicaine, en exaltant une identité « musulmane ». Le flou de cette catégorie permet à la fois de réactiver l'image de l'intégration républicaine, liée au modèle historique de développement de l'Etat, et caractérisée notamment par la centralisation et l'effacement des différences régionales et culturelles. Elle permet également de rappeler à l'ordre tout ce qui semble contester ce modèle de référence. Passé au prisme des dispositions antisubversives des agents des services de renseignement, le communautarisme devient de la sorte un ensemble cohérent, doté d'organisations centrales et avançant masqué, dont les finalités politiques doivent être mises en lumière. Interrogé par des parlementaires sur le foulard dit « islamique », Yves Bertrand (DCRG) expliquait de la sorte :

"A mon avis, ils testent nos capacités à réagir et cherchent d'autres domaines. (...) Je pense que des orientations sont données et, qu'au-delà de l'école, le monde du travail pris au sens large est maintenant visé, avec, comme cibles, certaines catégories de personnel. Ils ne vont pas s'en prendre, bien évidemment, aux ingénieurs. Pour l'instant, ils s'en prennent à des catégories de personnel plus modeste. Je pense, par exemple, aux manutentionnaires, (...) aux jeunes filles caissières dans les supermarchés ${ }^{46}$.

Menace violente pour la société ou danger pour la république, l'Islam fondamentaliste apparaît donc sous l'œil des services de renseignement comme problématique a priori. Pour eux, il cumule en effet une dimension trans-nationale (cohérent avec la manipulation étrangère), de fortes communautés implantées dans les Etats occidentaux, mais occupant des positions basses dans les hiérarchies sociales, et une idéologie hostile à l'ordre social et politique dominant. Cet Islam se présente de la sorte 
comme un projet global de subversion susceptible de se substituer au communisme, frappé par les restructurations du capitalisme post-fordiste et l'effondrement de l'URSS. Il prend sa place dans le discours de pouvoir et de normalisation décrit par Michel Foucault, et dans lequel la société «sera menacée par un certain nombre d'éléments hétérogènes, mais qui ne lui sont pas essentiels, qui ne partagent pas le corps social, le corps vivant de la société, en deux parties, mais qui sont en quelque sorte accidentels. Ce sera l'idée d'étrangers qui se sont infiltrés, ce sera le thème des déviants qui sont les sous-produits de cette société $»^{47}$. De là, les discours sur l'altérité irréductible des musulmans (comme hier, des classes dangereuses), la focalisation sur les convertis et le renforcement du contrôle policier.

La rencontre de cette suspicion généralisée avec les moyens de la lutte anti-terroriste a produit des effets néfastes pour les libertés individuelles, que le 11 septembre 2001 est encore venu aggraver. La France s'est dotée au moment des attentats de 1986 d'une législation spécifique en matière de lutte contre le terrorisme. Celle-ci repose sur la concentration des outils anti-terroristes entre les mains d'une section du parquet de Paris (14 ${ }^{\mathrm{ème}}$ section), d'un cabinet de juges d'instruction spécialisés et d'une unité de police judiciaire : la direction nationale anti-terroriste (DNAT). Cet ensemble cohérent vanté par les policiers pour son "efficacité " a déjà fait l'objet de très nombreuses critiques, tant de la part des magistrats que des défenseurs des droits de l'Homme ${ }^{48}$. Outre les problèmes liés à la centralisation, elles portent surtout sur l'usage systématique de deux incriminations floues - «l'association de malfaiteurs » et «en vue d'une entreprise terroriste »- dans les procédures. Ces incriminations permettent en effet d'utiliser ce que les professionnels appellent la stratégie du "filet», ou du " coup de pied dans la fourmilière ». Elle consiste à arrêter de manière extrêmement large des individus dont on pense qu'ils peuvent être liés d'une manière ou d'une autre à des réseaux radicaux. Elle repose sur la croyance en sa capacité à déstabiliser des réseaux, et à mettre à mal une logistique. Et peu lui importe si une bonne partie des prévenus est ensuite innocentée après avoir passé un ou deux ans en détention préventive. En règle générale, en matière de terrorisme "islamiste ", le rapport entre le nombre d'arrestations, le nombre d'inculpations et de culpabilités avérées apparaitt totalement disproportionné.

Les attentats du 11 septembre 2001, par l'émotion qu'ils ont suscitée et le prétexte qu'ils ont représenté, n'ont fait que renforcer ces procédures. En France, le vote successif de la loi relative à la sécurité quotidienne (31 octobre 2001), de la loi pour la sécurité intérieure (12 février 2003) et de la loi portant adaptation de la justice aux évolutions de la criminalité (11 février 2004) ont accru les pouvoirs de police et diminué le rôle de la défense. De la même manière, en Grande-Bretagne, la promulgation de l'Anti-terrorism, Crime and Security Act, en décembre 2001, a permis la mise en détention illimitée de personnes suspectes d'être des terroristes internationaux, sans que leur culpabilité n'ait été juridiquement établie (voir encadré ci-dessous).

\section{Du coupable au suspect}

La législation antiterroriste britannique adoptée en 2000 et modifiée en décembre 2001 (Anti-Terrorism Crime and Security Act) a permis l'incarcération illimitée, sans inculpation ni jugement d'étrangers soupçonnés de terrorisme, mais qui refusaient d'être expulsés, ou ne pouvaient l'être ${ }^{49} .17$ personnes ont de la sorte été 
emprisonnées - dont huit pendant trois ans - dans la prison de haute sécurité de Belmarsh, à Londres. Le 16 décembre 2004, ces mesures ont été déclarées illégales par les plus hauts magistrats britanniques. Les Law Lords les estimaient en effet contraire aux «instincts et aux traditions du peuple du Royaume-Uni» et discriminatoires, puisqu'elles ne touchent que les étrangers.

La réponse du gouvernement de Tony Blair ne se fit pas attendre. Le 26 janvier 2005, le ministre de l'Intérieur, Charles Clarke, abolit la distinction nationaux/ étrangers, en proposant que tous les individus soupçonnés par le Security Service (MI5), d'être ou d'avoir été lié à des activités terroristes, mais pour lesquels il n'existe pas de preuves recevables en justice soient soumis à une série de contrôles (Control Orders). Le suspect peut ainsi se voir imposer - pour une durée de 12 mois, renouvelable à volonté - un couvre-feu, une assignation à résidence, un "marquage" (un bracelet électronique) et des restrictions d'accès aux communications, comme Internet ou le téléphone. Toute violation de l'un de ces contrôles est considérée comme un délit et peut être punie d'une peine allant jusqu'à cinq ans d'emprisonnement.

La violente opposition des parlementaires à ce projet obligea le gouvernement à accepter que ces contrôles soient approuvés préalablement par un juge et ne relèvent pas simplement de la compétence des autorités politiques. Mais l'essentiel demeure. La revendication des députés de l'obligation d'avancer des preuves et pas de simples soupçons pour priver un individu de ses libertés fondamentales est restée lettre morte. Et c'est la philosophie qui sert de colonne vertébrale au Prevention of Terrorism Act adopté le 11 mars 2005. Elle n'est pas nouvelle. Elle marque déjà l'ensemble des mesures en vigueur pour lutter contre les comportements "anti-sociaux» des jeunesses populaires. Le Anti-Social Behaviour Order (ASBO) permet en effet d'imposer des restrictions, comme celles de fréquenter un quartier, d'emprunter les transports publics, d'utiliser un téléphone portable, etc. à des adolescents qui n'ont pas commis de délit, mais dont les comportements sont "susceptible d'effrayer, d'alarmer ou de tourmenter" le voisinage... Toute infraction à ces contrôles est punie par la loi, et le plus souvent par la prison. Ces contrôles sont si drastiques que plus d'un tiers des mesures prononcées jusqu'ici a donné lieu à des poursuites pénales...

La «guerre à l'incivilité » et la "guerre au terrorisme» du gouvernement empruntent ainsi la même voie : rendre la vie impossible à certains individus et les enfermer dès qu'ils contreviennent à l'encadrement draconien auquel ils sont soumis au quotidien. Ces sacrifices des libertés individuelles sont justifiés au nom de l'urgence et de l'ampleur des «menaces » qui pèseraient sur la société. Ils sont l'aboutissement d'un fantasme sécuritaire qui fait des suspects des coupables, dépourvus des droits élémentaires de se défendre et innocents jusqu'à ce que l'on ait pu prouver le contraire.

L'ensemble de ces mesures renforce le petit monde des professionnels de l'antiterrorisme et les transactions collusives ${ }^{50}$ qui les lient. C'est-à-dire qu'au-delà d'une stricte évolution du droit, elles confortent et légitiment un univers de pratiques profondément illibérales, mais peu contestées, qui constituent de véritables poches d'exceptionnalisme enchâssées au cœur des régimes libéraux. Yves Bertrand, l'ancien directeur des RG expliquait à ce sujet :

«Il existe toujours un conflit entre l'efficacité policière et le respect des libertés. Cela dit, la défense des libertés n'est invoquée que lorsque les attentats ont cessé depuis un certain temps. Dans les périodes d'attentats, tout le monde est heureux de profiter d'un dispositif policier et judiciaire (...) permettant de rétablir le calme $»^{51}$. 
Certes. Mais le sacrifice des libertés au nom de l'anti-terrorisme ne va pas de soi. Quels que soient les actes commis par un individu, c'est précisément la capacité à garantir une égalité juridique et une justice équitable qui fondent les Etats de droit. Le recours aux mesures, voire aux pratiques d'exception a toujours eu des conséquences funestes pour les sociétés qui y ont consenti. Roy Jenkins, le secrétaire d'Etat britannique responsable du Prevention of Terrorism Bill réinstaurant largement les pouvoirs d'exception en Irlande du Nord en 1974, indiquait de la sorte :

«A l'époque, comme tout le monde, je pensais que ces pouvoirs étaient justifiés, et je le pense encore. Mais je pensais qu'ils seraient temporaires et qu'au bout des deux ans prévus, on reviendrait à la normale, c'est-à-dire à la protection des libertés. Je suis horrifié maintenant de savoir que ces pouvoirs exceptionnels sont toujours en vigueur et si on me l'avait dit à l'époque j'aurais refusé de le croire, et si je l'avais cru, j'aurais refusé de les appliquer "52. $^{52}$.

Son confrère italien Francesco Cossiga, ministre de l'Intérieur lors des années de plomb signalait quant à lui :

«Je fais mon mea-culpa. On en est au culte de la délation, à la canonisation des collaborateurs de justice [les « repentis »]. Et c'est en partie de ma faute... Chaque soir je fais un acte de contrition pour avoir contribué, dans les années soixante-dix, à l'expansion de cette manière de rendre la justice. Alors j'étais en guerre, mais c'est là un cancer qui tue le système judiciaire. (...) La justice italienne est entièrement faite de rumeurs, bavardages, délations... Je pense présenter une proposition de loi pour changer les choses: Je prends les règles de l'Inquisition de Torquemada, et je les traduis en italien d'aujourd'hui. Il y a là plus de garanties que dans notre code de procédure pénale... 1 $^{53}$.

Ces opinions convergentes de promoteurs de mesures d'exception justifiées par la lutte contre le « terrorisme » sont à méditer. Elles sont hélas d'une criante actualité.

\section{NOTES}

1. En France, on pense au fameux « il faut terroriser les terroristes » prononcé par Charles Pasqua, lorsqu'il était ministre de l'Intérieur, au sujet des nationalistes corses.

2. MM. François Thuillier, Fernando Reinares et Antonio Diaz m'ont beaucoup éclairé sur les situations britannique et espagnole. Qu'ils en soient ici chaleureusement remerciés.

3. Sur les problèmes de sources concernant les services de renseignement, voir ma contribution au State of the art Challenge, accessible sur http://www.libertysecurity.org/

4. Dobry M., «Le renseignement dans les démocraties occidentales. Quelques pistes pour l'identification d'un objet flou ", Les cahiers de la sécurité intérieure, n³0, $4^{\mathrm{ème}}$ trimestre 1997, pp. 53-85.

5. Allison G.T., Essence of Decision, Explaining the Cuban Missiles Crisis, $2^{\text {ème }}$ éd., Longman, 1999.

6. Edelman M., Pièces et règles du jeu politique, Paris, Seuil, 1991, pp. 53 et suivantes.

7. Tilly C., « Terror, Terrorism, Terrorists ", Sociological Theory, 22/1, mars 2004, pp. 5-13.

8. "Position commune du Conseil du 27 décembre 2001 relative à l'application de mesures spécifiques en vue de lutter contre le terrorisme»(2001/931/PESC), Journal officiel des Communautés européennes, 28 décembre 2001, L 344/95-96. 
9. Les données sur la Corse sont issues du rapport de M. Raymond Forni, La sécurité: un droit pour les Corses, un devoir pour l'Etat, ( $\left.\mathrm{n}^{\circ} 1918\right)$, Assemblée nationale, novembre 1999.

10. Le Monde, 12 septembre 2002.

11. Audition devant la commission parlementaire du 11M, 18 novembre 2004.

12. Schelling T.C., Stratégie du conflit, Paris, PUF, 1986.

13. Sur ces processus, concernant les attentats de 1986 en France, voir Duclos L.-J., « Les pouvoirs publics et la campagne terroriste moyen-orientale : France 1986 », Etudes polémologiques, n49, 1/1989, pp. 75-109.

14. Keller W.W., The Liberals and J. Edgar Hoover. Rise and Fall of a Domestic Intelligence State, Princeton, Princeton University Press, 1989 ; et Gill P., Policing Politics. Security Intelligence and the Liberal Democratic State, Londres, Frank Cass Editor, 1994.

15. Lacroix B., « Pour une science politique réflexive. Enjeux et usages de la référence à Norbert Elias », Tumultes, $n^{\circ} 15$, octobre 2000, p. 195.

16. Dewerpe A., Espion. Une anthropologie historique du secret d'Etat contemporain, Paris, Gallimard, 1984, p. 14.

17. Voir notamment Marx G.T., Undercover. Police Surveillance in America, Berkeley, University of California Press, 1988.

18. Pour des descriptions par des anciens policiers des RG, voir par exemple Dufourg J.M., Section manipulation, de l'antiterrorisme à l'affaire Doucé, Paris, Laffont, 1991 ; et Rougelet P., RG, la machine à scandales, Paris, Albin Michel, 1997.

19. Voir sur ces sujets Guittet E.-P., Raison et déraison d'Etat : les GAL (Grupos Antiterroristas de Liberacion) 1983-1987, mémoire de DEA, Université Paris X-Nanterre, 2000 ; et Bigo D., Guittet E.-P. et Smith A., «La participation des militaires à la sécurité intérieure : Royaume Uni, Irlande du Nord ", Cultures et Conflits, $n^{\circ} 56,2004$, pp. 11-34.

20. Palidda S., Polizia postmoderna. Etnografia del nuovo controllo sociale, Milan, Feltrinelli, 2000.

21. Ces désaccords sur la nature de l'activité policière apparaissent clairement dans les commentaires portés sur les autres services. Un commissaire de sécurité publique fustigeait de la sorte l'activité de ses collègues des RG qu'il considérait comme une majorité « de feignants, dont l'activité essentielle est de fréquenter les réceptions et les cocktails» [Entretien, janvier 1999]. L'un de ses homologues des $\mathrm{RG}$ répondait quant à lui aux critiques de la police judiciaire sur les montées en généralité qu'il opérait dans les notes transmises à sa direction centrale en disant: "ils ne comprennent pas ce qu'on fait, maisla police judiciaire est de toute façon allergique aux concepts» [Entretien, mai 1999].

22. Sur les caractéristiques des champs, voir notamment Bourdieu P., «Quelques propriétés des champs ", in Questions de sociologie, Paris, Editions de minuit, 1984, pp. 113 et suivantes; et du même auteur, Esquisse d'une théorie de la pratique, Paris, Seuil 2000, pp. 25 et suivantes.

23. Bonelli L., "Formation, conservation et reconversion de dispositions antisubversives. L'exemple des renseignements généraux », in Tissot S. (dir.), Reconversions militantes, Limoges, Presses universitaires de Limoges, 2005.

24. Monjardet D., Ce que fait la police. Sociologie de la force publique, Paris, La Découverte, 1996, p. 151.

25. Audition de M. Yves Bertrand, directeur central des Renseignements Généraux, 9 juillet 2003, Rapport de M. Jean-Louis Debré sur la question du port des signes religieux à l'école, ( $\left.\mathrm{n}^{\circ} 1275\right)$ Assemblée nationale, décembre 2003. Nous soulignons.

26. Voir Rogin M., Les démons de l'Amérique, Paris, Seuil, 1998, pp. 17-18.

27. Dezcallar de Mazarredo J., «Lucha contra el terrorismo internacional », El noticiero de las ideas, n¹3, Enero-Marzo de 2003.

28. Mauss M., Sociologie et anthropologie, Paris, PUF, 1999 [1950], p. 89.

29. Johnson R., "Defending Ways of Life. The (Anti)-Terrorist Rhetorics of Bush and Blair ", Theory, Culture \& Society, 2002, vol.19, n4, pp. 211-231. 
30. El País, 28 avril 2002.

31. Bourdieu P., Propos sur le champ politique, Lyon, Presses Universitaires de Lyon, 2000, p. 63.

32. Khosrokhavar F., Les nouveaux martyrs d'Allah, Paris, Flammarion, 2003, p. 250.

33. "Global Terrorism: Are we meeting the challenge?", lecture at City of London Police Headquarters, 16 octobre 2003.

34. Dezcallar de Mazarredo J., « Lucha contra el terrorismo internacional », op. cit.

35. Manningham-Buller E., " Global Terrorism: Are we meeting the challenge? », op. cit.

36. Bigo D., «La recherche proactive et la gestion du risque », Déviance et Société, décembre 1997, vol. 21, n 4 , pp. 423-429.

37. Audition de M. Yves Bertrand, directeur central des Renseignements Généraux, 9 juillet 2003, Rapport de M. Jean-Louis Debré sur la question du port des signes religieux à l'école, op. cit.

38. Libération, 6 décembre 2002.

39. Rogin M., "La répression politique aux Etats-Unis », Actes de la recherche en sciences sociales, n²0, décembre 1997, pp. 32-44.

40. Voir Césari J., Musulmans et républicains. Les jeunes, l'islam et la France, Bruxelles, Complexe, 1998 ; et Sayad A., La double absence. Des illusions de l'émigré aux souffrances de l'immigré, Paris, Seuil, 1999.

41. Beaud S., Masclet O., « Un passage à l'acte improbable ? Notes de recherche sur la trajectoire sociale de Zacarias Moussaoui ", French Politics, Culture and Society, vol. 20, n², été 2002; Khosrokhavar F., Les nouveaux martyrs d'Allah, op. cit., pp. 271 et suivantes. Dans un autre registre, voir Leveau R., « Réflexions sur le non-passage au terrorisme dans l'immigration maghrébine en France », Etudes polémologiques, n49, 1/1989, pp. 141-156.

42. Voir notamment Huntigton S., The Clash of Civilizations and Remaking of World Order, New York, Simon and Schuster, 1996. Pour une critique radicale des conditions d'élaboration et de réception de cette thèse, voir le numéro spécial de Cultures \& Conflits, "Troubler et inquiéter. Les discours du désordre international », n¹9/20, automne-hiver 1995.

43. Césari J., Musulmans et républicains..., op. cit.

44. Bousquet R., Insécurité : les quartiers de tous les dangers, Paris, L'Harmattan, 1998, p. 151.

45. Par exemple, la revue militaire Le Casoar ( $n^{\circ} 142$, juillet 1996, p.24) décrivait ainsi la situation des quartiers à forte concentration de populations d'origine immigrée : «les liens conservés par ces immigrés avec leur pays d'origine peuvent les rendre sensibles aux appels de ces derniers à l'occasion de différends éventuels avec la notre. Les fameuses cinquièmes colonnes, évoquées à l'occasion de maints conflits passés seraient ainsi déjà en place ».

46. Audition de M. Yves Bertrand, directeur central des Renseignements Généraux, 9 juillet 2003, Rapport de M. Jean-Louis Debré sur la question du port des signes religieux à l'école, op. cit. Je souligne. 47. Foucault M., Il faut défendre la société. Cours au collège de France 1976, Paris, Gallimard-Seuil, 1997, p. 70.

48. Voir notamment Syndicat de la magistrature, «A quoi peuvent bien servir des juges antiterroristes ? ", Justice,n`146, novembre 1995, pp. 3-6 ; et Fédération Internationale des Ligues des Droits de l'Homme (FIDH), France: la porte ouverte à l'arbitraire. Rapport d'une mission internationale d'enquête en France sur l'application de la législation anti-terroriste, concernant particulièrement les conditions de détention provisoire et l'exercice des droits de la défense, Hors série de la Lettre bimensuelle de la FIDH, n 271, janvier 1999.

49. Guild E., «Agamben face aux juges. Souveraineté, exception et antiterrorisme », Cultures \& Conflits, L'Harmattan, n51, automne 2003, pp. 127-156.

50. Dobry M., Sociologie des crises politiques, Paris, FNSP, 1986, pp. 110 et suivantes.

51. Audition de M. Yves Bertrand directeur central des Renseignements Généraux, 29 juin 1999, Rapport de M. Raymond Forni, La sécurité : un droit pour les Corses, un devoir pour l'Etat, op. cit., p. 167.

52. Jenkins R., A Life at the Centre, Macmillan, Londres, 1991, p. 397 et suivantes. 

frappeur, 2005.

\section{RÉSUMÉS}

Après les attentats spectaculaires et meurtriers du 11 septembre 2001 aux Etats-Unis, puis ceux du 11 mars 2004 à Madrid, les services de renseignement occidentaux ont vu leur rôle et leur poids s'accroître dans la lutte contre le radicalisme musulman. A partir d'une enquête portant sur les services français, espagnols et britanniques, cet article analyse les évolutions de leurs missions et de leurs analyses de cette question. Rompant avec une approche mécaniste, qui verrait dans la transformation de ces missions le simple fruit d'une " adaptation » à de nouvelles menaces, il montre que leur nature et leur forme résultent à la fois de l'évolution de l'activité des groupes clandestins et de leur perception; des effets de la permanence des grilles d'analyse des services de renseignement qui avaient été forgées sur d'autres terrains; et du travail perpétuel de relégitimation que ces services vont mener auprès de certains secteurs de l'appareil d'Etat. Il contribue de la sorte à éclairer certains aspects souvent méconnus des modes pratiques de régulation de la violence politique dans les démocraties occidentales.

Following the spectacular and deadly September 11th, 2001 attacks in the US and the March 11th, 2004 attacks in Madrid, the western intelligence services have seen their role and weight increase in the fight against radical Islam. This text, based on a fieldwork on French, Spanish and British intelligence services aims at analysing the evolution of their missions and analysis of the question. Breaking with an overly-mechanical approach that would consider the transformation of these missions as the result of an "adaptation » to new threats, this text shows that their nature and form result both from the evolution of clandestine groups' activities and their perception; the effects of the continuity of their analysis grids and of the perpetual work of relegitimisation that they lead among certain parts of the State apparatus. This article thereby contributes to shedding light on specific aspects of the practical modes of political violence regulation in the western democracies that are often underrated.

\section{INDEX}

Mots-clés : Renseignement, exception, suspicion, ennemi, ELISE

\section{AUTEUR}

\section{LAURENT BONELLI}

Laurent BONELLI, Groupe d'Analyse Politique, Université Paris X-Nanterre. Il est chargé de recherches au Centre d'Etudes sur les Conflits et membre de l'équipe française des programmes européens ELISE et CHALLENGE. 